\title{
Estamos Preparados para Diagnosticar e Conduzir um Episódio de Hipertermia Maligna? *
}

\section{Are we Prepared to Diagnose and Managed Malignant Hyperthermia?}

Claudia Marquez Simões ${ }^{1}$, Giovanna Negrisoli Koishi ${ }^{2}$, Marcelo Rozatti ${ }^{3}$, José Luiz Gomes do Amaral, TSA ${ }^{4}$

\author{
RESUMO \\ Simões CM, Koishi GN, Rozatti M, Amaral JLG - Estamos Pre- \\ parados para Diagnosticar e Conduzir um Episódio de Hiperter- \\ mia Maligna?
}

\begin{abstract}
Justificativa e Objetivos - A Hipertermia Maligna é doença rara e potencialmente fatal associada à exposição a agentes halogenados e succinilcolina. A mortalidade e morbidade resultante pode ser reduzida pelo diagnóstico precoce e tratamento específico, o que inclui o dantrolene sódico. A população brasileira é de aproximadamente 160 milhões de habitantes assistidos por mais de 6000 anestesiologistas. $\mathrm{Na}$ última década, sobretudo, considerável atenção foi dada à esta doença, disto resultando especialistas melhor informados e mais hospitais aparelhados para trata-la. Este estudo visa avaliar o nível atual de informação acerca da Hipertermia Maligna entre os anestesiologistas brasileiros, de sorte a orientar novas iniciativas voltadas para o controle desta afecção.
\end{abstract}

Método - Vinte questões sobre diagnóstico, prevenção e tratamento da Hipertermia Maligna foram enviadas aos 6164 membros da Sociedade Brasileira de Anestesiologia (SBA).

Resultados - Seiscentos e quarenta e seis anestesiologistas $(10,4 \%)$ responderam às questões. Foram obtidas mais de $90 \%$ das respostas corretas sobre diagnóstico e tratamento. Por outro lado, aproximadamente $50 \%$ das respostas sobre indicação da biópsia muscular e farmacologia do dantrolene estavam erradas.

Conclusões - Os resultados acima refletem nível satisfatório de informação sobre este assunto, indicando alguns relevantes aspectos da doença que merecem atenção adicional. O número de respostas é significativo para avaliação do grau de conhecimento sobre este assunto, mas denota insuficiente motivação. Destes achados conclui-se ser necessário ampliar os esforços de educação continuada, contemplando todo os diferentes tópicos deste importante tema da Anestesiologia.

UNITERMOS: COMPLICAÇÕES: hipertermia maligna

\footnotetext{
* Recebido da (Received from) Disciplina de Anestesiologia, Dor e Terapia Intensiva da Universidade Federal de São Paulo, com apoio da Associação Acadêmica de Anestesiologia Dor e Terapia Intensiva da Faculdade de Medicina do $A B C$

1. $M E_{1}$ do CET/SBA da FMUSP, Membro da Secretaria da "Sempre Viva" Associação Brasileira de Combate a Hipertermia Maligna

2. ME Cirurgia Geral da Faculdade de Medicina do ABC

3. $M E_{2}$ do CET/SBA da UNIFESP, Membro do Conselho Fiscal da "Sempre Viva" - Associação Brasileira de Combate a Hipertermia Maligna

4. Professor Titular da Disciplina de Anestesiologia, Dor e Terapia Intensiva da UNIFESP
}

Apresentado (Submitted) em 14 de maio de 2002

Aceito (Accepted) para publicação em 03 de outubro de 2002

Correspondência para (Mail to):

Dra. Cláudia Marquez Simões

Pça. Antonio Manuel do Espírito Santo, 63 - Jardim Bonfiglioli 05592-050 São Paulo, SP

(C) Sociedade Brasileira de Anestesiologia, 2003

\author{
SUMMARY \\ Simões CM, Koishi GN, Rozatti M, Amaral JLG - Are we Prepa- \\ red to Diagnose and Managed Malignant Hyperthermia?
}

Background and Objectives - Malignant Hyperthermia (MH) is an uncommon but potentially lethal disease associated to halogenate agents and/or succinylcholine exposure. Resulting mortality and morbidity may be decreased by early diagnosis and specific treatment, including sodium dantrolene. Brazil has approximately 160 million inhabitants assisted by more than 6000 anesthesiologists. In the last decade, special attention was given to this disease, resulting in better informed anesthesiologists and more prepared hospitals to treat malignant hyperthermia (MH). This study aimed at measuring the level of information of Brazilian anesthesiologists regarding $\mathrm{MH}$, in order to develop new approaches to control this disease.

Methods - A questionnaire with 20 questions on malignant hyperthermia diagnosis, prevention and treatment was mailed to the 6,164 members of the Brazilian Society of Anesthesiology.

Results - Questionnaires were returned by 646 anesthesiologists (10.4\%). More than $90 \%$ of correct answers about clinical diagnosis and treatment were obtained. On the other hand, nearly $50 \%$ of anesthesiologists gave incorrect answers about muscle biopsy indication and clinical pharmacology of dantrolene.

Conclusions - Results have indicated adequate knowledge about this topic, but has shown that some relevant issues need additional attention. The number of answers was significant to evaluate $\mathrm{MH}$ understanding of Brazilian anesthesiologists, but has also shown poor motivation. From these results it is possible to conclude that it is essential to intensify continuing education programs, contemplating all issues of this major anesthetic subject.

KEY WORDS: COMPLICATIONS: malignant hyperthermia

\section{INTRODUÇÃO}

Aipertermia Maligna (HM) é afecção hereditária e latenAte, caracterizada por resposta hipermetabólica aos anestésicos voláteis, (halotano, isoflurano, sevoflurano e desflurano) e succinilcolina ${ }^{1}$. Em humanos a HM é herança dominante, provavelmente associada a mais de um gene, nem todos os susceptíveis compartilhando do mesmo padrão genético ${ }^{2}$. Ainda que diferentes tecidos possam apresentar alterações, a HM é considerada doença primária do músculo esquelético ${ }^{3,4}$. As crises de HM devem-se a desarranjo da homeostase intracelular do cálcio e se expressam por hiperatividade contrátil, hipertermia, aumento do consumo de oxigênio e da produção de gás carbônico, acúmulo de ácido lático, desacoplamento da fosforilação oxidativa, lise celular e extravazamento do conteúdo do citoplasma. Entre as manifestações iniciais de HM encontram-se taquicardia, rigidez muscular, instabilidade hemodinâmica, taquipnéia e cianose ${ }^{5}$. A elevação da temperatura pode ser tardia. Ela é assinalada como manifestação inicial em ape- 
nas $30 \%$ dos casos ${ }^{5}$. A elevação da pressão parcial de gás carbônico no sangue arterial $\left(\mathrm{PaCO}_{2}\right)$ e na mistura exalada $\left(\mathrm{P}_{\mathrm{ET}} \mathrm{CO}_{2}\right)$ é encontrada precocemente em um episódio de $\mathrm{HM}$, refletindo o hipermetabolismo, característico da doença ${ }^{6}$. A presença desses sinais em paciente exposto a agentes desencadeantes exige imediata consideração da possibilidade de HM. Os passos fundamentais para o controle de uma crise de HM vêm a ser interrupção da exposição aos agentes desencadeantes (substituindo-os por agentes seguros) e correção da alteração do metabolismo celular de cálcio com dantrolene sódico. O tratamento de complicações como hipertermia, acidose metabólica e respiratória, disritmias cardíacas, disfunção renal e distúrbios da coagulação incluem hiperventilação, bicarbonato venoso, resfriamento ativo, antiarrítmicos, expansão volêmica e diuréticos ${ }^{7}$. Dada a potencial evolução fulminante e letal, o diagnóstico precoce e o tratamento específico são de crucial importância, permitindo redução da mortalidade de $70 \%$ para menos de $10 \%^{8}$.

O objetivo deste estudo é avaliar o nível de informação que os anestesiologistas brasileiros dispõem acerca da HM, sorte a estimar sua capacidade em diagnosticar e tratar adequadamente um episódio desta doença.

\section{MÉTODO}

Este estudo foi projeto conjunto da Liga de Anestesiologia da Faculdade de Medicina do ABC e da Disciplina de Anestesiologia, Dor e Terapia Intensiva da UNIFESP, contando com a colaboração da Sociedade Brasileira de Anestesiologia, que, após analisar a proposição, facilitou o contato com os anestesiologistas brasileiros.

O projeto baseou-se em questionário elaborado de modo a abranger os aspectos mais importantes do diagnóstico e tratamento da HM (Anexol), enviado para os 6.164 anestesiologistas membros da SBA, em junho de 1999. As questões foram apresentadas em envelope contendo cartão para respostas pré-postado. Acrescentou-se à correspondência um segundo envelope contendo as respostas comentadas, com o intuito de aproveitar o momento para também oferecer informação sobre o assunto (Anexo II). Os resultados foram processados e analisados em conjunto e independentemente para cada uma das regionais da SBA.

\section{RESULTADOS}

Seiscentos e quarenta e seis anestesiologistas, ou seja, $10,4 \%$ dos membros da SBA, responderam ao questionário. A distribuição de respondedores entre as Regionais da SBA encontra-se na tabela I. Dentre as regionais da SBA, a que apresentou maior número de respondedores foi a de Santa Catarina, com participação de $21 \%$ de seus membros, seguida pelo Rio Grande do Sul com $18,32 \%$ de respostas. Não houve participação dos membros das regionais dos estados do Amapá, Acre, Roraima e Rondônia.
Tabela I - Participação dos Anestesiologistas no Questionário sobre Hipertermia Maligna, nos Diferentes Estados da Federação

\begin{tabular}{|c|c|c|}
\hline Regional da SBA & $\begin{array}{l}\text { Número de } \\
\text { respondedores }\end{array}$ & $\begin{array}{c}\% \text { Respondedores da } \\
\text { Regional }\end{array}$ \\
\hline Santa Catarina & 28 & 21,21 \\
\hline Rio Grande do Sul & 70 & 18,32 \\
\hline Piauí & 06 & 14,63 \\
\hline Sergipe & 08 & 14,03 \\
\hline Minas Gerais & 72 & 13,08 \\
\hline Tocantins & 03 & 13,04 \\
\hline São Paulo & 210 & 12,18 \\
\hline Paraná & 43 & 12,07 \\
\hline Alagoas & 05 & 11,1 \\
\hline Espírito Santo & 10 & 10,41 \\
\hline Rio de Janeiro & 87 & 8,99 \\
\hline Mato Grosso & 02 & 8,69 \\
\hline Goiás & 18 & 7,96 \\
\hline Paraíba & 07 & 7,6 \\
\hline Pernambuco & 22 & 6,6 \\
\hline Bahia & 18 & 6,38 \\
\hline Amazonas & 04 & 6,06 \\
\hline Mato Grosso do Sul & 04 & 5,63 \\
\hline Ceará & 12 & 4,95 \\
\hline Pará & 05 & 4,9 \\
\hline Maranhão & 02 & 4,44 \\
\hline Rio Grande do Norte & 03 & 3,94 \\
\hline Distrito Federal & 07 & 3,64 \\
\hline Acre & 0 & 0 \\
\hline Amapá & 0 & 0 \\
\hline Roraima & 0 & 0 \\
\hline Rondônia & 0 & 0 \\
\hline
\end{tabular}

Foram obtidas mais de $90 \%$ das respostas certas sobre diagnóstico e tratamento. Por outro lado, aproximadamente 50\% das respostas sobre indicação da biópsia muscular e farmacologia do dantrolene estavam incorretas. Os índices de acerto por temas estão no Anexo II.

\section{DISCUSSÃO}

Descrita na primeira metade dos anos $60^{\circ}$, a HM passou a ser melhor compreendida nas duas décadas seguintes. As bases para seu diagnóstico e tratamento consolidaram-se a partir de 1980. Em 1992 já existia a preocupação em difundir informações sobre HM no Brasil ${ }^{10}$. Desde 1990, nos Congressos Brasileiros de Anestesiologia, nas Jornadas e em 
outros eventos regionais, tem-se procurado divulgar as bases clínicas da HM. Ao mesmo tempo estabeleceu-se plantão de informações sobre HM (Hot-Line), Cadastro de Pacientes (Universidade Federal de Santa Catarina), Centros de Diagnóstico de Hipertemia Maligna (Universidade Federal do Rio de Janeiro e Universidade de São Paulo) e a Sempre Viva, Associação Brasileira de Combate à Hipertermia Maligna (fundada por pacientes susceptíveis e seus familiares). Este notável trabalho tornou a HM conhecida por praticamente toda a comunidade de anestesiologistas do país. Em 1999, mais de 100 biópsias musculares haviam sido registradas no Brasil (Sudo RT, informação pessoal).

Até a década de 90 não era possível importar regularmente o dantrolene sódico, há vários anos já adotado na América do Norte e Europa, como o único tratamento específico para HM. Raras instituições dispunham do medicamento, adquirido em bases informais. Apenas em 1996 foi autorizada a importação regular de dantrolene sódico. Em 1997, 22 regionais da SBA receberam doação de 36 frascos com $20 \mathrm{mg}$ de dantrolene sódico e praticamente todos os Estados do Brasil passaram a contar com este medicamento.

O limitado percentual de respondedores sugere que ainda existe longo caminho a percorrer antes que se alcance plena consciência da importância deste grave problema. Por outro lado, o índice de acerto mostra qualificação satisfatória do anestesiologista brasileiro. Em geral o índice de acerto foi maior nos Estados de Espírito Santo (85\% de acertos), Mato Grosso do Sul ( $80 \%$ ) Pernambuco e Bahia (75\% de acertos). Nos demais Estados registrou-se índices de acerto de 60 a $70 \%$.

Entre os respondedores, encontra-se bom nível de conhecimento sobre diagnóstico e tratamento. Observa-se, todavia, uma grande porcentagem de respostas equivocadas quando as perguntas versavam sobre biópsia muscular ou farmacologia do dantrolene sódico. O resultado deste estudo permite direcionar os esforços de divulgação sobre HM entre os anestesiologistas brasileiros. Assim, parece razoável supor que maior ênfase seja dada à veiculação de informações sobre biópsia muscular e dantrolene sódico.

Apesar do exposto, o dantrolene ainda não é encontrado em muitas instituições de saúde brasileiras. Esta talvez seja uma das razões do desconhecimento acerca de suas propriedades farmacológicas. Até o início do ano 2000 o dantrolene era disponível em 54 hospitais brasileiros, distribuído em 47 cidades, em 20 dos 26 estados brasileiros. No início de 2002, 305 hospitais, distribuídos em 142 cidades, em 24 dos 26 estados brasileiros já possuíam este medicamento (A relação dos hospitais que possuem o medicamento pode ser encontrada no site da SBA, http://www.sba.com.br, na seção sobre Hipertermia Maligna).

Observou-se que muitas instituições não possuem a dose mínima recomendada de dantrolene sódico para interromper as manifestações de um episódio agudo de Hipertermia Maligna. Em certas regiões os hospitais dividem esta dose mínima. A partir de março de 2002, por força da Lei Paulo Teixeira e do Decreto Geraldo Alckmin as entidades de assistência a saúde do Estado de São Paulo deverão garantir o tratamento específico imediato dos pacientes que vierem a apresentar HM, responsabilizando-se civil e criminalmente pela eventual omissão ${ }^{11}$. Espera-se que esta obrigação estenda-se em futuro próximo aos demais Estados da Federação. As autoridades de saúde devem estar cientes sobre a escassez de recursos para o tratamento de episódios de HM e tomar medidas urgentes visando garantir a disponibilidade de dantrolene sódico em todos os hospitais do Brasil.

Conclui-se do exposto estar o Brasil ainda distante do ideal para o controle da HM, mas vê-se que a especialidade caminha decididamente na direção certa. O limitado número de respostas entre os anestesiologistas brasileiros e as lacunas em alguns aspectos relevantes da HM indicam que este tema deve ser continuamente enfatizado nos programas de educação continuada. 


\begin{tabular}{|c|c|}
\hline \multicolumn{2}{|c|}{ ANEXO I - QUESTIONÁRIO ENVIADO AOS MEMBROS DA SOCIEDADE BRASILEIRA DE ANESTESIOLOGIA } \\
\hline $\begin{array}{l}\text { Assinale a resposta correta: } \\
\text { Questão } 1 . \\
\text { A Hipertermia Maligna é definida como: } \\
\text { a) Afecção hereditária e latente, caracterizada por resposta hipermetabólica aos anestési- } \\
\text { cos voláteis e à succinilcolina; } \\
\text { b)Hipertermia progressiva e miólise maciça desencadeadas por estresse; } \\
\text { c)Doença do sistema nervoso central com acometimento secundário do músculo esquelé- } \\
\text { tico; } \\
\text { d)Elevacão súbita e acentuada da temperatura corporal. }\end{array}$ & $\begin{array}{l}\text { Questão } 11 . \\
\text { Os seguintes anestésicos e agentes são considerados "seguros" no que concerne a hiper- } \\
\text { termia maligna: } \\
\text { a)Óxido nitroso, halotano, etomidato; } \\
\text { b)Sevoflurano, óxido nitroso, opióides; } \\
\text { c)Cetamina, atracúrio, propofol; } \\
\text { d)Opióides, succinilcolina, cetamina. } \\
\text { Resposta [ ] }\end{array}$ \\
\hline Resposta [ ] & $\begin{array}{l}\text { Questão } 12 \text {. } \\
\text { A administracão profilática de dantrolene sódico é indicada em: }\end{array}$ \\
\hline $\begin{array}{l}\text { Questão } 2 . \\
\text { São agentes capazes de desencadear uma crise de Hipertermia Maligna: } \\
\text { a)Halotano, succinilcolina e propofol; } \\
\text { b)Succinilcolina, enflurano e sevoflurano; } \\
\text { c)Atracúrio, isoflurano e droperidol; } \\
\text { d)Sevoflurano, etomidato e bupivacaína. } \\
\text { Resposta [ ] }\end{array}$ & $\begin{array}{l}\text { a)História pregressa de rigidez de masseter; } \\
\text { b)Pacientes com síndrome neuroléptica maligna; } \\
\text { c)Disfunção cardiocirculatória ou renal graveque torne o paciente incapaz de tolerar a fase } \\
\text { inicial de um episódio de Hipertermia Maligna; } \\
\text { d)Gestantes com história familiar de hipertermia desencadeada por estresse. } \\
\text { Resposta [ ] }\end{array}$ \\
\hline & Questão 13. \\
\hline $\begin{array}{l}\text { Questão } 3 \text {. } \\
\text { Com relação à epidemiologia da Hipertermia Maligna, é correto afirmar que: } \\
\text { a)A incidência varia em virtude da exposição aos agentes desencadeantes e à freqüência } \\
\text { dos genes determinantes nas diferentes populações; } \\
\text { b)Incide a cada } 50000 \text { anestesias regionais; } \\
\text { c)É mais rara em crianças; } \\
\text { d)As crises ocorrem em igual freqüência, tanto em homens como em mulheres. } \\
\text { Resposta [l }\end{array}$ & $\begin{array}{l}\text { Com relação ao dantrolene sódico podemos afirmar que: } \\
\text { a)Age na modulação da concentração extracelular de cálcio; } \\
\text { b)É um antitérmico inespecífico; } \\
\text { c)O descenso da temperatura após sua administração serve para confirmar diagnóstico } \\
\text { de hipertermia maligna; } \\
\text { d)Não tem efeitos colaterais. } \\
\text { Resposta [ ] }\end{array}$ \\
\hline & Questão 14. \\
\hline $\begin{array}{l}\text { Questão } 4 . \\
\text { A crise de Hipertermia Maligna deve-se a um desarranjo do metabolismo intracelular do } \\
\text { cálcio e está associada a: } \\
\text { a)Hiperatividade contrátil, hipertermia e acidemia; } \\
\text { b)Hiperatividade contrátil, alcalose e hipertermia; } \\
\text { c)Hipoatividade contrátil, hipertermia e acidemia; } \\
\text { d)Hipoatividade contrátil, hipertermia e alcalemia. }\end{array}$ & $\begin{array}{l}\text { Em indivíduos susceptíveis, a prevenção da Hipertermia maligna inclui: } \\
\text { a)Evitar exposição a agentes desencadeantes; } \\
\text { b)Monitorizar a temperatura central e a capnografia; } \\
\text { c)Monitorizar pressão venosa central e pressão capilar pulmonar; } \\
\text { d)Administrar dantrolene antes da indução da anestesia. } \\
\text { Resposta [ ] }\end{array}$ \\
\hline Resposta [ ] & $\begin{array}{l}\text { Questão } 15 . \\
\text { A Hipertermia Maligna está relacionada com: }\end{array}$ \\
\hline $\begin{array}{l}\text { Questão } 5 \text {. } \\
\text { Em ordem decrescente de incidência, as manifestações iniciais de uma crise de hiperter- } \\
\text { mia são: } \\
\text { a)Taquícardia, rigidez muscular, hipertermia; } \\
\text { b)Hipertermia, taquicardia e cianose; } \\
\text { c)Hipertermia mioglobinemia e taquicardia: }\end{array}$ & $\begin{array}{l}\text { a)Rigidez de masseter, síndrome de King-Denborough, doença de Crohn; } \\
\text { b)Síndrome neuroléptica maligna, diabetes mellitus, hipertensão arterial sistêmica } \\
\text { c) Síndrome King Denborough, rigidez de masseter, central core disease } \\
\text { d)Distrofia muscular, esclerose múltipla, central core disease } \\
\text { Resposta [ ] }\end{array}$ \\
\hline mioglobinúria e instabilidade hemodinâmica. & Ques \\
\hline Resposta [ ] & $\begin{array}{l}\text { Dentre os principais efeitos colaterais do dantrolene sódico temos: } \\
\text { a)Flebite, náusea, hipertensão arterial; }\end{array}$ \\
\hline $\begin{array}{l}\text { Questão } 6 . \\
\text { Qual dos recursos de monitorização traz maior precocidade no diagnóstico de uma crise } \\
\text { de Hipertemia Maligna? } \\
\text { a)Termômetro; } \\
\text { b)Cardioscópio. }\end{array}$ & $\begin{array}{l}\text { b) Vômitos, atonia uterina, flebite; } \\
\text { c)Diarréia, vômitos, flebite; } \\
\text { d)Hipopotassemia, vômitos, flebite. } \\
\text { Resposta [ ] }\end{array}$ \\
\hline c)Oxímetro de & Questão 17. \\
\hline $\begin{array}{l}\text { d)Capnógrafo. } \\
\text { Resposta [] }\end{array}$ & $\begin{array}{l}\text { Sobre Hipertermia Maligna é correto dizer que: } \\
\text { a)A simples elevação da temperatura é essencial para o diagnóstico de Hipertermia Malig- } \\
\text { na; }\end{array}$ \\
\hline $\begin{array}{l}\text { Questão } 7 . \\
\text { O tratamento de uma crise de Hipertermia Maligna Maligna inclui: } \\
\text { a)Interrupção da administração do anestésico volátil halogenado, hiperventilação e inje- } \\
\text { ção venosa de dantrolene sódico ( } 2 \text { a } 2,5 \mathrm{mg} \cdot \mathrm{kg}^{-1} \text {, repetidos até o controle das manifesta- } \\
\text { cões); } \\
\text { b)Administração de dantrolene sódico ( } 1 \mathrm{mg} \cdot \mathrm{kg}^{-1} \text {, venoso) a cada } 6 \text { horas, resfriamento ati- } \\
\text { vo e bicarbonato de sódio: }\end{array}$ & $\begin{array}{l}\text { b)É relevante um antecedente familiar de primeiro grau; } \\
\text { c)Toda pessoa susceptível desenvolve Hipertermia Maligna quando exposta aos agentes } \\
\text { desencadeantes; } \\
\text { d)O aumento dos níveis sangüíneos de creatino-fosfocinase (CPK) faz parte do diagnósti- } \\
\text { co precoce da Hipertermia Maligna. } \\
\text { Resposta [ ] }\end{array}$ \\
\hline $\begin{array}{l}\text { c) Resfriamento ativo e controle das disritmias, pois a Hipertermia Maligna é auto-limitada; } \\
\text { d)Interrupção da administração de agentes desencadeantes, injeção venosa de dantrole- } \\
\text { ne }\left(2 \mathrm{mg} \cdot \mathrm{kg}^{-1}\right) \text { e antagonistas de canais de cálcio. } \\
\text { Resposta [ ] }\end{array}$ & $\begin{array}{l}\text { Questão } 18 . \\
\text { Oprincipal dado deanamnese para identificarum portador do traço para susceptibilidade à } \\
\text { Hipertermia Maligna é: } \\
\text { a)Presença de algum familiar em que tenha sido diagnosticado Hipertermia Maligna; }\end{array}$ \\
\hline $\begin{array}{l}\text { Questão } 8 . \\
\text { As principais complicações da Hipertermia Maligna são: } \\
\text { a)Hipertensão arterial, insuficiência hepática, hiperglicemia e glicosúria; } \\
\text { b)Insuficiência respiratória aguda, hipopotassemia, hipercalcemia e atrofia muscular; }\end{array}$ & $\begin{array}{l}\text { b)Manifestações de febre e cansaço muscular freqüente; } \\
\text { c)Manifestaços de cãimbra e fraqueza; } \\
\text { d)Hipertireoidismo e distrofia muscular. } \\
\text { Resposta [ ] }\end{array}$ \\
\hline $\begin{array}{l}\text { c) Hipotermia, hipocalcemia, hipermagnesemia e coagulação intravascular disseminada; } \\
\text { d)Choque, disritmias cardíacas, insuficiência renal e coagulação intravascular dissemi- } \\
\text { nada. } \\
\text { Resposta [ ] }\end{array}$ & $\begin{array}{l}\text { Questão } 19 \text {. } \\
\text { Doze horas após uma hepatectomia sob anestesia balanceada (isoflurano e fentanil), o } \\
\text { paciente, até então sem alterações clínicas, vem a apresentar aumento da temperatura, } \\
\text { taquicardia, taquipnéia e hipercarbia. Pode-se concluir que: }\end{array}$ \\
\hline $\begin{array}{l}\text { Questão } 9 . \\
\text { Após o diagnóstico clínico de um episódio de Hipertermía Maligna é necessário: } \\
\text { a)Informar verbalmente e por escrito o paciente e seus familiares da situação, detalhando } \\
\text { os riscos envolvidos; } \\
\text { b)Restringir a atividade física em clima quente; } \\
\text { c)Recomendar a administração profilática de dantrolene em caso de outra anestesia; } \\
\text { d)Contra-indicar qualquer tipo de anestesia no futuro. } \\
\text { Resposta [ ] }\end{array}$ & $\begin{array}{l}\text { a)A Aassociação das manifestações descritas conduz ao diagnóstico de Hipertermia Malig- } \\
\text { na; } \\
\text { b)Não é provável tratar-se de Hipertermia Maligna, pois já há muito tempo foi interrompida } \\
\text { a exposição ao isoflurano; } \\
\text { c)O dantrolene sódico deve ser administrado imediatamente; } \\
\text { d)Exclui-se o diagnóstico de hipertermia Maligna face a ausência de antecedentes familia- } \\
\text { res. } \\
\text { Resposta [ ] }\end{array}$ \\
\hline $\begin{array}{l}\text { Questão } 10 . \\
\text { Com relação à biópsia muscular, pode-se afirmar que: } \\
\text { a)Está indicada em todos os pacientes em que foi diagnosticada uma crise de Hipertermia } \\
\text { Maligna, bem como nos casos de rigidez de masseter; } \\
\text { b)Os músculos preferidos paraarealização dabiópsia são o glúteo máximo e o deltóide; } \\
\text { c)Deve ser realizada imediatamente após uma crise suspeita de Hipertermia Maligna; } \\
\text { d)Todos os familiares de um paciente suspeito de susceptibilidade devem ser igualmente } \\
\text { submetidos à biópsia muscular. } \\
\text { Resposta [ ] }\end{array}$ & $\begin{array}{l}\text { Questão 20. O controle da Hipertermia Maligna baseia-se em: } \\
\text { a)Difusão do conhecimento acerca dos diferentes aspectos desta doença; } \\
\text { b)Monitorização adequada (incluindo capnografia); } \\
\text { c)Disponibilidade imediata de tratamento específico (dantrolene sódico); } \\
\text { d)Identificação dos susceptíveis (biópsia muscular); } \\
\text { e)Todas as anteriores estão corretas. } \\
\text { Resposta [ ] }\end{array}$ \\
\hline
\end{tabular}


RESPOSTAS E COMENTÁRIOS

Consulte o gabarito apenas depois de ter preenchido a folha de respostas.

Questão 1.(96,75\% acertos)

Resposta A

AHipertermia Maligna é afecção hereditária e latente caracterizada por resposta hipermetabólica aos anestésicos voláteis (como halotano, enflurano, isoflurano, sevoflurano e desflurano) e à succinilcolina.

\section{Questão 2.(82,66\% acertos)}

Resposta B

Àexceção da succinilcolina e os anestésicos voláteis como o halotano, enflurano, isoflurano, sevoflurano e desflurano, os demais agentes empregados em anestesia são considerados seguros e não se associam a Hipertermia Maligna.

\section{Questão 3.(88,24\% acertos)}

Resposta A

Espera-se maior número de casos quando anestésicos voláteis e succinilcolina forem mais usados. Tratando-se de doença hereditária é possivel encontrar populações em que os genes envolvidos ocorram com maior freqüência.

A Hipertermia Maligna é encontrada em caucasianos e orientais. As crises são raras em A Hipertermia Maligna e encontrada em caucasianos e orientais. As crises são raras em extremos de idade e mais comuns em crianças, sobretudo aquelas entre 8 e 12 anos. Ain-
da que a herança não seja ligada ao sexo, as crises são mais encontradas em homens que da que a heranç

\section{Questão 4.(95,36\% acertos)}

\section{Resposta A}

Em uma crise de Hipertermia Maligna, em resposta à exposição aos agentes desencadeantes (anestésicos voláteis e/ou succinilcolina) a elevação dos níveis de cálcio intracel lar determinam hiperatividade contrátil, aumento da atividade das bombas iônicas dependentes de energia, aumento do consumo de oxigênio e produção de gás carbônico, hipertermia, anaerobiose, acidemia, destruição celular, mioglobinemia e mioglobiunúria.

\section{Questão 5.(78,17\% acertos) \\ Resposta A}

A hipercapnia, detectada à capnografia é manifestação precoce da Hipertermia Maligna, refletindo assim o hipermetabolismo (produção aumentada de gás carbônico) associadoà síndrome. Taquicardia e rigidez muscular surgem, nesta ordem de freqüência, antes de outras manifestações. A elevação da temperatura é observada como manifestação inicial em apenas cerca de $30 \%$ das crises de Hipertermia Maligna.

\section{Questão 6.(77,41\% acertos)}

Resposta D

Acapnografia permite identificar com precocidade uma crise de Hipertermia Maligna. Recomenda-se usá-la sempre que for empregado um agente desencadeante (AAssociação Americana de Anestesiologia - Eichhorn JH: ASA Newsletter March 1999 6[3]:14-15. - definiu em 1999, que a capnografia está indicada sempre que realizada anestesia geral, em qualquer de suas formas). O cardioscópio e a oximetria de pulso também são úteis, pois taquicardia é uma das manifestações iniciais da Hipertermia Maligna. Atermometria é útil, porém a elevação da temperatura pode ser uma manifestação tardia, em um episódio de Hipertermia Maligna.

\section{Questão 7.(90,56\% acertos)}

\section{Resposta A}

No tratamento de uma crise de Hipertermia Maligna recomenda-se interrupção da exposição aos agentes desencadeantes, hiperventilação (controle da hipercapnia) com oxigênio puro e injeção intravenosa de dantrolene sódico em injeções de 2 a $2,5 \mathrm{mg} \cdot \mathrm{kg}^{-1}$, repetidas até o controle das manifestações clínicas. A acidose metabólica pode ser atenuad com bicarbonato de sódio. As arritmias cardíacas podem estar associadas a hiperpotassemia. Busca-se controlar a elevação do potássio plasmático através da hiperventilação, da alcalinização com bicarbonato de sódio ou administração de solução contendo glicose e insulina. No tratamento das arritmias está contra-indicado administrar antagonistas de canais de cálcio, visto que estes agentes estão associados a colapso circulatório na presença de dantrolene sódico. O resfriamento ativo (compressas frias, irrigação gástrica, vesical ou peritoneal com solução salina resfriada) auxilia a controlar a hipertermia.

\section{Questão 8.(88,08\% acertos) \\ Resposta D}

A instabilidade hemodinâmica é, caracteristicamente, manifestação inicial de uma crise de Hipertermia Maligna. Ela se manifesta através de taquicardia, arritmias e hipertensão inicial, que rapidamente evolui para hipotensão e choque. Ainsuficiência renalé o resultado da associação de hipoperfusão renal e deposição tubular de mioglobina. Complicação das crises gravesé a coagulação intravascular disseminada, problema de difícil controle.

\section{Questão 9.(96,29\% acertos)}

\section{Resposta A}

É absolutamente necessário oferecer ao susceptível a Hipertermia Maligna e seus familiares toda a informação a respeito deste problema. Trata-se de cuidado essencial para a prevenção de episódios semelhantes. Pais e irmãos de susceptíveis têm $50 \%$ de probabilidade de também o serem. Aos susceptíveis recomenda-se evitar sua exposição a agentes desencadeantes. Eles podem beneficiar-se de anestesia em procedimentos cirúrgicos ou odontológicos, desde que halogenados ou succinilcolina não estejam incluídos. Devido a seus efeitos colaterais adversos, o dantrolene não é usado profilaticamente.

\section{Questão 10.(43,19\% acertos)}

Resposta A

A biópsia muscular (preferencialmente o músculo vasto lateral) é usada para confirmar a susceptibilidade aos agentes desencadeantes e é realizada nos casos em que foi diagnosticado uma crise de Hipertermia Maligna. Cerca de $50 \%$ dos pacientes que tiveram rigidez de masseter são positivos para Hipertermia Maligna, à biópsia muscular. As biópsias são realizadas em pacientes com mais de 20 quilos e a não menos de 3 meses As boiópsias são realizadas em pacientes com mais de 20 quilos e a não menos de 3 meses depois de uma crise suspeita. Habitualmente são biopsiados os pais e irmãos dos casos
demonstrados (através de biópsia) positivos ( $50 \%$ de probabilidade de positividade). A demonstrados (através de biópsia) positivos ( $50 \%$ de probabilidade de positividade). A partir
lia.

\section{Questão $11(83,59 \%$ acertos)}

Resposta C

Todos os agentes usados em anestesia são seguros, exceção feita aos anestésicos voláteis - halotano, enflurano, isoflurano, sevoflurano e desflurano e à succinilcolina.

\section{Questão 12.(30,34\% acertos)}

Resposta C

Dada a improbabilidade de episódios graves de Hipertermia Maligna sem prévia exposição a agentes desencadeantes e o dantrolene não ser isento de efeitos colaterais, sua administração profilática é restrita a situações excepcionais como história pessoal pregressa de Hipertermia Maligna desencadeada por estresse; disfunção cardiocirculatória ou renal que torne o paciente incapaz de tolerar a fase inicial de um episódio de Hipertermia Maligna.

\section{Questão 13.(23,54\% acertos)}

Resposta B

dantrolene sódico restaura a homeostasia intracelular do cálcio. Ele é um antitérmico inespecífico, sorte que a defervescência após sua administração não permite concluir pela presença de Hipertermia Maligna. Entre os efeitos colaterais do dantrolene sódico, encontram-se flebite, náuseas, vômitos, atonia uterina, potencialização de bloqueio neuromuscular e, em interação com antagonistas de canais de cálcio, hiperpotassemia e colapso cardiovascular.

\section{Questão 14. (89,01\% acertos)}

Resposta A

Previne-se uma crise de Hipertermia Maligna eliminando halogenados e succinilcolina da anestesia de pacientes susceptíveis ou suspeitos de susceptibilidade.

Em virtude de seus efeitos colaterais adversos, não é recomendado o uso profilático de dantrolene sódico. Amonitorização da capnografia permite o diagnóstico precoce de uma crise de Hipertermia Maligna, porém não impede sua eclosão.

\section{Questão 15.(47,06\% acertos)}

Resposta C

São associados à Hipertermia Maligna, a Síndrome King Denborough, a rigidez de masseter e a miopatia conhecida como Central Core Disease. Distrofias musculares estão relacionadas a reações anormais graves à succinilcolina (hiperpotassemia, rabdomiólise, parada cardíaca).

\section{Questão 16.(38,41\% acertos)}

Resposta B

Além de náuseas, vômitos, atonia uterina e flebite, o dantrolene sódico associa-se a potencialização de bloqueio neuromuscular e, em interação com antagonistas de canais de cálcio, hiperpotassemia e colapso cardiovascular.

\section{Questão $17(58,98 \%$ acertos)}

Resposta B

Pais, filhos e irmãos de portadores do traço para Hipertermia Maligna têm $50 \%$ de probabilidade de também o serem, pois trata-se de herança dominante não ligada ao sexo. É necessário salientar que o diagnóstico definitivo de susceptibilidade é dado pela resposta ao teste halotano/cafeína (através de biópsia muscular). O diagnóstico de uma crise de $\mathrm{Hi}$ pertermia Maligna é clínico e fundamenta-se na associação das manifestações clínicas em paciente exposto a agentes desencadeantes. Isoladamente as manifestações de $\mathrm{Hi}$ pertermia Maligna não permitem diagnóstico, visto serem comuns a diversas outras situações freqüentes em anestesia. A hipertermia não se evidencia no quadro inicial de muitas crises de Hipertermia Maligna e os níveis de CPK alcançam valores máximos horas após o episódio.

\section{Questão 18.(93,98\% acertos)}

Resposta A

A hereditariedade é caraterística da Hipertermia Maligna. Entretanto, raras são as ocasiões em que essa informação é encontrada à anamnese. Por outro lado, os susceptiveis são, habitualmente, indivíduos assintomáticos, que têm vida absolutamente normal, não surgindo a Hipertermia Maligna senão quando ocorre exposição aos agentes desencadeantes.

\section{Questão $19 .(24,46 \%$ acertos $)$}

Resposta B

É improvável que uma crise de Hipertermia Maligna se manifeste depois de 3 horas de interrupção da exposição a um dos agentes desencadeantes.

\section{Questão 20.(95,36\% acertos)}

Resposta E

C controle da Hipertermia Maligna depende da consciência e mobilização dos suceptíveis e dos anestesiologistas em torno desta questão. Seremos nós que conduziremos nossos legisladores e a administração pública e privada a aparelharem as Instituições de Saúde para diagnosticar e tratar adequadamente esta doença. ASempre Viva é uma associação voltada para o combate à Hipertermia Maligna, que congrega susceptíveis e seus familiares, anestesiologistas e todos os interessados em controlar esta afecção. Junte-se a nós! 


\section{Are we Prepared to Diagnose and Managed Malignant Hyperthermia?}

Claudia Marquez Simões, M.D., Giovanna Negrisoli Koishi, M.D., Marcelo Rozatti, M.D., José Luiz Gomes do Amaral, TSA, M.D.

\section{INTRODUCTION}

Malignant Hyperthermia $(\mathrm{MH})$ is an inherited and latent disease, characterized by hypermetabolic response to volatile anesthetics (halothane, isoflurane, sevoflurane and desflurane) and succinylcholine ${ }^{1}$. In humans, $\mathrm{MH}$ is a dominant heritage, probably associated to more than one gene, and not all susceptible individuals share the same genetic pattern ${ }^{2}$. While different tissues may be affected, $\mathrm{MH}$ is considered a primary skeletal muscle disease ${ }^{3,4}$. MH crises are triggered by a disorder in intracellular calcium homeostasis and are expressed by contractile hyperactivity, hyperthermia, increase in oxygen consumption and $\mathrm{CO}_{2}$ production, lactic acid accumulation, oxidative phosphorilation decoupling, cell lysis and cytoplasm content leakage. Tachycardia, muscle stiffness, hemodynamic instability, tachypnea and cyanosis are among early $\mathrm{MH}$ manifestations ${ }^{5}$. Hyperthermia may be a late episode and is described as initial manifestation in only $30 \%$ of cases. $\mathrm{CO}_{2}$ partial pressure increase in arterial blood $\left(\mathrm{PaCO}_{2}\right)$ and in exhaled mixture $\left(\mathrm{P}_{\mathrm{ET}} \mathrm{CO}_{2}\right)$ is early found in $\mathrm{MH}$, reflecting the hypermetabolic characteristic of such disease ${ }^{6}$. The presence of those signs in patients exposed to triggering agents requires immediate consideration of $\mathrm{MH}$. Fundamental steps to control $\mathrm{MH}$ are: interruption of triggering agents exposure (replacing them by safe agents) and correction of intracellular calcium metabolic changes with sodium dantrolene. Complications, such as hyperthermia, metabolic and respiratory acidosis, arrhythmias, renal dysfunction and coagulation disorders may be treated with hyperventilation, intravenous bicarbonate, active cooling, antiarrhythmic drugs, volume expansion and diuretics ${ }^{7}$. Given the potential lethal evolution, early diagnosis and specific treatment are critical and may decrease mortality from $70 \%$ to less than $10 \%{ }^{8}$.

This study aimed at evaluating the level of information of Brazilian anesthesiologists about $\mathrm{MH}$ to estimate their ability to adequately diagnose and treat such disease.

\section{METHODS}

This study was jointly designed by the Anesthesiology League, Faculdade de Medicina do ABC and by the Discipline of Anesthesiology, Pain and Intensive Care, UNIFESP, with the cooperation of the Brazilian Society of Anesthesiology (SBA) which, after analyzing the proposition, has helped the contact with Brazilian anesthesiologists.

The project was based on a questionnaire designed to comprehend all major issues of $\mathrm{MH}$ diagnosis and treatment (Attachment I), which was mailed to all 6,164 anesthesiologists members of SBA, in June 1999. Questions were sent in an envelope with a pre-posted answering card. A second envelope was added to the correspondence with commented answers, aiming at using the moment to also give information about the subject (Attachment II). Results were jointly processed and analyzed in separate for each SBA regional section.

\section{RESULTS}

Answers were returned by 646 anesthesiologists, or $10.4 \%$ of SBA members. Table I shows the distribution of respondents among SBA regional sections. Santa Catarina was the regional section with more answers $(21 \%)$, followed by Rio Grande do Sul (18.32\%). Amapá, Acre, Roraima and Rondonia members have not participated.

Table I - Participation of Anesthesiologists in the Questionnaire About Malignant Hyperthermia, in Different Brazilian States

\begin{tabular}{|c|c|c|}
\hline SBA Regional Section & $\begin{array}{l}\text { Number of } \\
\text { Respondents }\end{array}$ & $\begin{array}{l}\% \text { of regional } \\
\text { respondents }\end{array}$ \\
\hline Santa Catarina & 28 & 21.21 \\
\hline Rio Grande do Sul & 70 & 18.32 \\
\hline Piauí & 06 & 14.63 \\
\hline Sergipe & 08 & 14.03 \\
\hline Minas Gerais & 72 & 13.08 \\
\hline Tocantins & 03 & 13.04 \\
\hline São Paulo & 210 & 12.18 \\
\hline Paraná & 43 & 12.07 \\
\hline Alagoas & 05 & 11.1 \\
\hline Espírito Santo & 10 & 10.41 \\
\hline Rio de Janeiro & 87 & 8.99 \\
\hline Mato Grosso & 02 & 8.69 \\
\hline Goiás & 18 & 7.96 \\
\hline Paraíba & 07 & 7.6 \\
\hline Pernambuco & 22 & 6.6 \\
\hline Bahia & 18 & 6.38 \\
\hline Amazonas & 04 & 6.06 \\
\hline Mato Grosso do Sul & 04 & 5.63 \\
\hline Ceará & 12 & 4.95 \\
\hline Pará & 05 & 4.9 \\
\hline Maranhão & 02 & 4.44 \\
\hline Rio Grande do Norte & 03 & 3.94 \\
\hline Distrito Federal & 07 & 3.64 \\
\hline Acre & 0 & 0 \\
\hline Amapá & 0 & 0 \\
\hline Roraima & 0 & 0 \\
\hline Rondônia & 0 & 0 \\
\hline
\end{tabular}

More than $90 \%$ correct answers about diagnosis and treatment were obtained. On the other hand, approximately $50 \%$ of answers about muscle biopsy indication and dantrolene 
pharmacology were incorrect. Rightness rates by subject are shown in Attachment II.

\section{DISCUSSION}

Described in the first half of the $60 \mathrm{~s}^{9}$, $\mathrm{MH}$ became better understood in the following 2 decades. Bases for diagnosis and treatment were consolidated as from 1980. in 1992 there was already a concern in spreading information about $\mathrm{MH}$ in Brazil $^{10}$. Since 1990, clinical MH bases are being presented in Brazilian Congresses of Anesthesiology, Journeys and other regional events. At the same time, there are institutions such as MH Hot-Line, Patients' Files (Federal University of Santa Catarina), Malignant Hyperthermia Diagnostic Centers (Federal University of Rio de Janeiro and University of São Paulo), and "Sempre Viva", a very active Brazilian Association for Malignant Hyperthermia Prevention (founded by susceptible patients and their relatives). This wonderful work made $\mathrm{MH}$ known by virtually all members of the Brazilian community of anesthesiologists. In 1999, more than 100 muscle biopsies had been recorded throughout Brazil (Sudo $\mathrm{RT}$, personal information).

Until the 90s, it was impossible to regularly import sodium dantrolene, already adopted for a long time in the USA and Europe as the only specific $\mathrm{MH}$ treatment. Few institutions had the drug informally acquired. Only in 1996 the regular import of sodium dantrolene was authorized. In 1997, 22 SBA regional sections received a donation of 36 vials with $20 \mathrm{mg}$ sodium dantrolene and almost all Brazilian states started to count on this drug.

The small percentage of respondents suggests that there is still a long way to go before full awareness of this severe problem is achieved. On the other hand, the percentage of right answers shows an adequate qualification of Brazilian anesthesiologists. In general, rightness rate was higher in the states of Espírito Santo (85\%), Mato Grosso do Sul $(80 \%)$, Pernambuco and Bahia $(75 \%)$. Remaining states had rightness rates of $60 \%$ to $70 \%$.

There has been an adequate understanding of diagnosis and treatment among respondents. There was, however, a major percentage of incorrect answers about muscle biopsy and sodium dantrolene pharmacology. Results of this study have allowed the redirection of efforts to spread $\mathrm{MH}$ information among Brazilian anesthesiologists. So, it seems reasonable to assume that more emphasis should be given to information about muscle biopsy and sodium dantrolene.

In spite of the above, sodium dantrolene is still not available in several Brazilian health institutes. This might be one of the reasons for the lack of knowledge about its pharmacological properties. Until early 2000 , dantrolene was available in 54 Brazilian hospitals, distributed through 47 cities, in 20 out of 26 Brazilian states. In early 2002, 305 hospitals distributed through 142 cities, in 24 out of 26 Brazilian states had this drug available (the list with the hospitals having the drug may be found in SBA's website, http://www.sba.com.br under Malignant Hyperthermia).

It was observed that several institutions do not have the minimum recommended sodium dantrolene dose to interrupt the manifestations of an acute Malignant Hyperthermia episode. In some regions, hospitals split this minimum dose. As from March 2002, under law Paulo Teixeira and Decree Geraldo Alckmin, health institutes of the state of São Paulo shall assure immediate specific treatment to $\mathrm{MH}$ patients, being civil and legally responsible for any omission ${ }^{11}$. Hopefully this duty will be expanded in the near future to all Brazilian states. Health authorities should be aware of the lack of resources to treat $\mathrm{MH}$ episodes and take urgent measures to assure the availability of sodium dantrolene in all Brazilian hospitals. The conclusion from the above is that Brazil is still far from ideal in controlling $\mathrm{MH}$, but the specialty is steadily walking in the right direction. The limited number of answers and the gaps in some relevant $\mathrm{MH}$ issues indicate that the subject should be continuously emphasized in continuing education programs.

\section{REFERÊNCIAS - REFERENCES}

01. Denborough M - Malignant Hyperthermia. Lancet, 1998;352:1131-1136.

02. Lerman J, Sims C - Anesthesia and muscle diseases. Adv Anesth, 1996;13:247-269.

03. Iaizzo PA, Kehler CH, Carr RJ et al - Prior hypothermia attenuates malignant hyperthermia in susceptible swine. Anesth Analg, 1996;82:803-809.

04. Roewer N, Dziadzka A, Greim CA et al - Cardiovascular and metabolic responses to anesthetic-induced malignant hyperthermia in swine. Anesthesiology, 1995;83:141-159.

05. Britt B - Malignant Hyperthermia, em: Orkin FK, Cooperman LH Complications in Anesthesiology. Philadelphia; JB Lippincott, 1983;291-313

06. Maccani RM, Wedel DJ, Hofer R - Norepinephrine does not potentiate porcine malignant hyperthermia. Anesth Analg, 1996;82:790-795.

07. Kaplan R - Malignant hyperthermia. Refresher Course Lectures. 1991 ASA Annual Meeting. San Francisco, 1991;231:1-7.

08. Britt B - Dantrolene. Can Anaesth Soc J, 1984;31:61-75.

09. Denborough M, Foster JFA, Lowell RRH et al. - Anaesthetic deaths in a family. Br J Anaesth, 1962;34:395-396.

10. Almeida Neto MA - Hipertermia maligna - Retrato brasileiro. Rev Bras Anestesiol, 1992;42:395-396.

11. Decreto $n^{\circ} .46 .601$ - Política Estadual de Prevenção Diagnóstico e Tratamento da Hipertermia Maligna. Diário Oficial do Estado de São Paulo, 12 de março de 2002. 
ATTACHMENT I - QUESTIONNAIRE MAILED TO MEMBERS OF THE BRAZILIAN SOCIETY OF ANESTHESIOLOGY

Choose the right answer:

Question 1

Malignant Hyperthermia is defined as:

a)Inherited and latent infection, characterized by hypermetabolic response to volatile anesthetics and succinylcholine;

b)Progressive hyperthermia and massive myolysis triggered by stress;

c) Central nervous system disease with secondary involvement of the skeletal muscle;

d) Sudden and severe body temperature increase.

Answer [ ]

Question 2

Agents able to trigger Malignant Hyperthermia crisis:

a) Halothane, succinylcholine and propofol:

b) Succinylcholine, enflurane and sevoflurane;

c)Atracurium, isoflurane and droperidol;

d) Sevoflurane, etomidate and bupivacaine.

Answer [ ]

Question 3

According to Malignant Hyperthermia epidemiology, one may state that:

a)Incidence varies with the exposure to triggering agents and with the frequency of determinant genes in different populations:

b) Occurs at every 50000 regional anesthesias:

c)ls less common in children

d) Crises are equally frequent in males and females.

Answer [ ]

Question 4.

Malignant Hyperthermia crisis is caused by intracellular calcium metabolic disorder and is assoMalignant
ciated to:

ractile hyperactivity, hyperthermia and acidemia;

b) Contractile hyperactivity, alkalosis and hyperthermia;

c)Contractile hypoactivity, hyperthermia and acidemia;

d) Contractile hypoactivity, hyperthermia and alkalemia.

Answer [ ]

Question 5

In decreasing order of incidence, early manifestations of malignant hyperthermia are:

a)Tachycardia, muscle stiffness, hyperthermia;

b) Hyperthermia, tachycardia and cyanosis;

c) Hyperthermia, myoglobinemia and tachycardia

d) Hyperthermia, myoglobinemia and hemodynamic instability.

Answer [ ]

Question 6

Which monitoring device provides the earliest diagnosis of Malignant Hyperthermia:

a)Thermometer;

b) Cardioscope;

c) Pulse oximeter;

d) Capnograph

Answer [ ]

Question 7 .

The treatment of Malignant Hyperthermia crisis includes:

a)Interruption of halogenate volatile anesthetics, hyperventilation and intravenous sodium dan-

trolene injection ( 2 to $2.5 \mathrm{mg} . \mathrm{kg}^{-1}$ repeated until manifestations control);

b) Sodium dantrolene administration (intravenous $1 \mathrm{mg}^{\mathrm{kg}}{ }^{-1}$ ) every 6 hours, active cooling and sodium bicarbonate;

c)Active cooling and arrhythmia control because Malignant Hyperthermia is self-limited;

d)Interruption of triggering factors administration, intravenous dantrolene $\left(2 \mathrm{mg} \cdot \mathrm{kg}^{-1}\right)$ and calci-

um channel antagonists.

Answer [ ]

Question 8.

Major Malignant Hyperthermia complications are:

a) Hypertension, liver failure, hyperglycemia and glycosuria;

b)Acute respiratory failure, hyponatremia and disseminated intravascular coagulation

c) Hypothermia, hypocalcemia, hypermagnesemia and disseminated intravascular coagulation; d) Shock, arrhythmias, renal failure and disseminated intravascular coagulation.

Answer [ ]

Question 9

After clinical diagnosis of Malignant Hyperthermia, it is necessary to:

a)Inform patients and relatives, orally and in writing, about the situation with details of involved

risks;

b)Limit physical activity in warm weather conditions;

c) Recommend prophylactic dantrolene administration in case of future anesthesia;

c) Recommend prophylactic dantrolene administration in

Answer [ ]

Question 10.

About muscle biopsy, it is right to state that:

a)It is indicated to all patients with Malignant Hyperthermia diagnosis, as well as in cases of masseter stiffness;

b)Preferred muscles for biopsy are gluteus maximus and deltoid;

c)It s should be immediately performed after a suspected Malignant Hyperthermia crisis;

d)All relatives of a patient with suspected susceptibility should be equally submitted to muscle biopsy.

Answer [ ]
Question 11.

The following anesthetics and agents are considered "safe" for Malignant Hyperthermia: a) Nitrous oxide, halothane, etomidate;

b) Sevoflurane, nitrous oxide, opioids;

c)Ketamine, atracurium, propofol;

d) Opioids, succinylcholine, ketamine

Answer [ ]

Question 12 .

Prophylactic sodium dantrolene administration is recommended in the presence of

a)Previous Masseter stiffness history;

b) Patients with malignant neuroleptic syndrome;

c) Severe cardiovascular or renal dysfunction making the patient unable to tolerate the initial stage of an acute Malignant Hyperthermia episode;

d)Pregnant women with family history of stress-triggered hyperthermia.

Answer [ ]

Question 13 .

About sodium dantrolene, it is correct to state that:

a) Acts in modulating extracellular calcium concentration;

b) Is a nonspecific antithermic agent:

c) The decrease in temperature after its administration is useful to confirm the diagnosis of malignant hyperthermia;

d) Has no side-effects.

Answer [ ]

Question 14

In susceptible individuals, Malignant Hyperthermia prevention consists of:

a)Avoiding exposure to triggering agents;

b)Monitoring central temperature and capnography;

c)Monitoring central venous pressure and pulmonary capillary pressure;

d)Administering dantrolene before anesthetic induction.

Answer [ ]

Question 15.

Malignant Hyperthermia is related to:

a)Masseter stiffness, King-Denborough syndrome, Crohn's disease;

b)Malignant neuroleptic syndrome, diabetes mellitus, systemic hypertension;

c)King-Denborough syndrome, Masseter stiffness, central core disease;

c)King-Denborough syndrome, Masseter stiffness, central core

d)Muscle dystr
Answer [ ]

Question 16

Among major sodium dantrolene side-effects:

a)Phlebitis, nausea, hypertension;

b)Vomiting, uterine atonia, phlebitis;

c)Diarrhea, vomiting, phlebitis;

d) Hyponatremia, vomiting phlebitis

Answer [ ]

Question 17.

About Malignant Hyperthermia, it is correct to state that:

a)A simple temperature increase is essential for the diagnosis of Malignant Hyperthermia;

b)A first degree relative history is relevant:

c) Every susceptible person will develop Malignant Hyperthermia when exposed to triggering agents:

d)Increased creatinine- phosphokinase (CPK) levels are part of early Malignant Hyperthermia diagnosis.

Answer [ ]

Question 18

Major anamnesis data to identify carriers of susceptibility traits to Malignant Hyperthermia are: a)Some relative diagnosed with Malignant Hyperthermia;

b)Frequent manifestations of fever and muscle weakness;

c)Manifestations of cramps and weakness;

d) Hyperthyroidism and muscle dystrophy.

Answer [ ]

Question 19

Twelve hours after hepatectomy under balanced anesthesia (isoflurane and fentanyl), patient otherwise without clinical complications, presents temperature increase, tachycardia, tachypnea and hypercarbia. One may conclude that:

a) The association of described manifestations leads to the diagnosis of Malignant Hyperthermia; b)It is unlikely to be Malignant Hyperthermia because isoflurane had already been withdrawn for a long time;

c)Sodium dantrolene should be immediately administered

d)Malignant Hyperthermia is ruled out due to the lack of family history.

Answer [ ]

Question 20 .

Malignant Hyperthermia control is based on:

a)Spread of knowledge about the different aspects of this disease;

b)Immediate availability of specific treatment (sodium dantrolene);

c)Identification of susceptible individuals (muscle biopsy);

d)All the above are correct.

Answer [ ] 


\section{ATTACHMENT II - COMMENTED ANSWERS TO THE QUESTIONNAIRE AND RIGHTNESS RATE BY QUESTION}

ANSWERS AND COMMENTS

Check the template only after having filled the answering card.

\section{Question 1. (96.75\% right)}

\section{Option A}

Malignant hyperthermia is an inherited and latent disease characterized by hypermetabolic response to volatile anesthetics (such as halothane, enflurane, isoflurane, sevoflurane and desflurane) and succinylcholine.

\section{Question 2. (82.66\% right)}

\section{Option $\mathrm{B}$}

With the exception of succinylcholine and volatile anesthetics such as halothane, enflurane, isoflurane, sevoflurane and desflurane, other anesthetic agents are considered safe and not related to Malignant Hyperthermia.

\section{Question 3. (88.24\% right)}

Option A

A larger number of cases is expected when volatile anesthetics and succinylcholine are more widely used. For being an inherited disease, it is possible to find populations where involved genes are more frequent.

Malignant Hyperthermia is found in Caucasians and Asians. Crises are uncommon in age extremes and more common in children, especially between 8 and 12 years of age. Although heritage not being related to gender, crises are more common in males as compared to females.

\section{Question 4. (95.36\% right)}

Option A

In a crisis of Malignant Hyperthermia in response to triggering agents exposure (volatile anesthetics and/or succinylcholine) intracellular calcium levels increase determines contractile hyperactivity, increased activity of energy-dependention pumps, increased oxygen consumption and $\mathrm{CO}_{2}$ production, hyperthermia, anaerobiosis, acidemia, cell destruction, myoglobinemia and myoglobinuria.

\section{Question 5. (78.17\% right)}

Option A

Hypercapnia detected by capnography is an early manifestation of Malignant Hyperthermia, thus reflecting the hypermetabolic state (increased $\mathrm{CO}_{2}$ production) associated to the syndrome. Tachycardia and muscle stiffness appear in this order of frequency, before other manifestations. Temperature increase is seen as initial manifestation in only approximately $30 \%$ of Malignant Hyperthermia episodes.

\section{Question 6 ( $77.41 \%$ right $)$}

\section{Option D}

Capnography allows the early detection of Malignant Hyperthermia. It is recommended whenever a triggering agent is used (The American Association of Anesthesiology - Eichhorn JH: ASA Newsletter March 1999 6[3]:14-15 - has defined in 1999 that capnography is indicated whenever general anesthesia is induced, with any technique). Cardioscope and pulse oximetry are also useful because tachycardia is an early Malignant Hyperthermia manifestation. Thermometry is useful, but temperature increase may be a late manifestation in Malignant Hyperthermia.

\section{Question 7 (90.56\% right)}

Option A

In treating Malignant Hyperthermia, it is recommended to interrupt the exposure to triggering agents, hyperventilate (hypercapnia control) with pure oxygen and administration of intravenous 2 to $2.5 \mathrm{mg} . \mathrm{kg}^{-1}$ sodium dantrolene, repeated until clinical manifestations are controlled. Metabolic acidosis may be attenuated with sodium bicarbonate. Arrhythmias may be associated to hypernatremia. Plasma potassium increase is controlled by hyperventilation, alkalinization with sodium bicarbonate or administration of glucose and insulin-containing solutions. Calcium channel antagonists are counterindicated to treat arrhythmias since those agents are associated to circulatory collapse in the presence of sodium dantrolene. Active cooling (cold compresses, gastric, vesical or peritoneal irrigation with cooled saline solution) helps controlling hyperthermia.

\section{Question 8. (88.08\% right)}

Option D

Hemodynamic instability is typically an early manifestation of Malignant Hyperthermia. It is manifested by tachycardia, arrhythmias and initial hypertension which rapidly progresses to hypotension and shock. Renal failure is the result of the association of renal hypoperfusion and myoglobin tubular deposition. Disseminated intravascular coagulation is a severe complication which is difficult to control.

\section{Question 9 (96.29\% right)}

Option A

It is absolutely necessary to give susceptible individuals and their relatives all information about the problem. It is an essential care to prevent similar episodes. Parents and sibling of susceptible individuals have $50 \%$ chance of being also susceptible. To susceptible individuals, it is recommended to avoid exposure to triggering agents. They may benefit from anesthesia in surgical or dental procedures, provided halogenate and succinylcholine are not included. Due to adverse side-effects, dantrolene is not prophylactically used.

\section{Question 10. (43.19\% right)}

Option A

Muscle biopsy (especially vastus lateralis muscle) is used to confirm susceptibility to triggering agents and is performed in cases where Malignant Hyperthermia has been diagnosed. Approximately $50 \%$ of patients with masseter stiffness are positive for Malignant Hyperthermia at muscle biopsy.

Biopsies are performed in patients weighing more than 20 kilos and not before 3 months after a suspected crisis. In general, parents and siblings of proven cases (by biopsy) are also submitted to biopsies ( $50 \%$ chance of being positive). After the results, other family members are referred to investigation.

\section{Question 11 (83.59\% right)}

Option C

All anesthetic agents are safe, except for volatile anesthetics - halothane, enflurane, isoflurane, sevoflurane and desflurane - and succinylcholine.

\section{Question 12 (30.34\% right)}

Option C

Due to the unlikelihood of severe Malignant Hyperthermia episodes without previous exposure to triggering agents, and the fact that dantrolene has side-effects, its prophylactic administration is limited to exceptional situations, such as previous personal history of stress-triggered Malignant Hyperthermia, cardiocirculatory or renal dysfunction making the patient unable to tolerate the initial stage of Malignant Hyperthermia.

\section{Question 13 (23.54\% right)}

Option B

Sodium dantrolene restores intracellular calcium homestasis. It is a nonspecific antithermic agent and the decrease in temperature after its administration does not allow for the diagnosis of Malignant Hyperthermia. Among sodium dantrolene side-effects, there are phlebitis, nausea, vomiting uterine atonia, neuromuscular block potentiation and in the presence of calcium channel antagonists, hypernatremia and cardiovascular collapse.

\section{Question 14 (89.01\% right)}

Option A

Malignant Hyperthermia is prevented by eliminating halogenates and succinylcholine from the anesthesia of patients susceptible or suspicious of Malignant Hyperthermia. Due to adverse side-effects, prophylactic sodium dantrolene administration is not recommended. Capnographic monitoring allows for the early diagnosis of Malignant Hyperthermia, however does not prevent its appearance.

\section{Question 15. (47.06\% right)}

Option C

King Denborough syndrome, masseter stiffness and the myopathy known as Central Core Disease are associated to Malignant Hyperthermia. Muscle dystrophies are related to severe abnormal reactions to succinylcholine (hypernatremia, rabdomyolysis, cardiac arrest)

\section{Question 16. (38.41\% right)}

Option B

In addition to nausea, vomiting uterine atonia and phlebitis, sodium dantrolene is associated to neuromuscular block potentiation and, in the presence of calcium channel antagonists, it is related to hypernatremia and cardiovascular collapse.

\section{Question 17 (58.98\% right)}

\section{Option B}

Parents, children and siblings of those with traits of Malignant Hyperthermia have $50 \%$ chance of being also susceptible because it is a dominant inherited disease unrelated to gender. It is necessary to stress that final susceptibility diagnosis is given by response to the halothane/caffeine test (through muscle biopsy). The diagnosis of a crisis of Malignant Hyperthermia is clinical and is based on the association of clinical manifestations in patients exposed to triggering agents. Malignant Hyperthermia manifestations per se do not allow a diagnosis because they are common to different anesthetic situations. Hyperthermia is not present in early manifestations of several Malignant Hyperthermia crises and PK reaches peak levels hours after the episode.

\section{Question 18 (93.98\% right)}

\section{Option A}

Heritage is characteristic of Malignant Hyperthermia. However, few are the cases in which such information is found during anamnesis. On the other hand, susceptible patients are in general asymptomatic individuals, with a perfectly normal life who do not present Malignant Hyperthermia unless they are exposed to triggering agents.

\section{Question 19 (24.46\% right)}

Option B

It is unlikely for a Malignant Hyperthermia crisis to manifest 3 hours after triggering agents exposure withdrawal.

\section{Question 20 (95.36\% right)}

Option E

Malignant Hyperthermia control depends on the awareness and mobilization of susceptible individuals and anesthesiologists around this subject. We will be the ones leading legislators and public and private administration to equip Health Institutes to adequately diagnose and treat this disease. "Sempre Viva" is an association aimed at fighting Malignan Hyperthermia, which gathers susceptible individuals and their families, anesthesiologists and all those interested in controlling this affection. Join us! 


\section{RESUMEN}

Simões CM, Koishi GN, Rozatti M, Amaral JLG - Estamos Preparados para Diagnosticar y Conducir un Episodio de Hipertermia Maligna?

Justificativa y Objetivos - La Hipertermia Maligna es una enfermedad rara y potencialmente fatal asociada a la exposición de agentes halogenados y succinilcolina. La mortalidad y morbidad resultante puede ser reducida por el diagnóstico precoz y tratamiento específico, lo que incluye el dantrolene sódico. La población brasileña es de aproximadamente 160 millones de habitantes asistidos por más de 6000 anestesiologistas. En la última década, sobre todo, considerable atención fue dada a esta enfermedad, de esto, resultando especialistas mejor informados y más hospitales con instrumentos necesarios para tratarla. Este estudio visa evaluar el nivel actual de información acerca de la Hipertermia Maligna entre los anestesiologistas brasileños, de suerte a orientar nuevas iniciativas orientadas para el control de esta afección.
Método - Veinte cuestiones sobre diagnóstico, prevención y tratamiento de la Hipertermia Maligna fueron enviadas a los 6164 miembros de la Sociedad Brasileña de Anestesiología (SBA).

Resultados - Seis cientos y cuarenta y seis anestesiologistas $(10,4 \%)$ respondieron a las cuestiones. Fueron obtenidas más de $90 \%$ de las respuestas correctas sobre diagnóstico y tratamiento. Por otro lado, aproximadamente $50 \%$ de las respuestas sobre indicación de la biopsia muscular y farmacología del dantrolene estaban erradas.

Conclusiones - Los resultados arriba reflejan nivel satisfactorio de información sobre este asunto, indicando algunos aspectos relevantes de la enfermedad que merecen atención adicional. El número de respuestas es significativo para evaluación del grado de conocimiento sobre este asunto, más denota insuficiente motivación. De estas notas se concluye que es necesario ampliar los esfuerzos de educación continuada, contemplando todo los diferentes tópicos de este importante tema de la Anestesiología. 